\title{
Fluorescent zinc oxide nanoparticles of Boswellia ovalifoliolata for selective detection of picric acid
}

\section{Geetha Venkatesan, Rajagopalan Vijayaraghavan*, Sarada Nallani Chakravarthula, Govindasamy Sathiyan}

Department of Chemistry, School of Advanced Sciences, VIT University, Vellore-632014, Tamil Nadu, India

*Corresponding Author. Email: rvijayaraghavan@vit.ac.in

Received: 29 March 2019, Accepted: 22 April 2019, Published Online: 25 April 2019

Citation Information: Geetha Venkatesan, Rajagopalan Vijayaraghavan, Sarada Nallani Chakravarthula, Govindasamy Sathiyan. Frontier Research Today 2019;2:2002. doi: 10.31716/frt.201902002 Cite in Other Styles

ABSTRACT: The use of Boswellia ovalifoliolata bark extract for the synthesis of zinc oxide nanoparticles (ZnO NPs) with benefits of eco-friendliness, low cost and compatibility for pharmaceutical and biological applications. In this paper, we report the biosynthesis of zinc oxide nanoparticles using plant extract and its application for the detection of picric acid (PA). The synthesized ZnO nanoparticles were characterized using UV-Vis spectroscopy, scanning electron microscopy (SEM), X-ray diffraction pattern (XRD), fourier transform infrared spectroscopy (FT-IR) and fluorescene spectroscopy. ZnO NPs in aqueous solution shows maximum absorption bands at $278 \mathrm{~nm}$ and fluorescence emission at $317 \mathrm{~nm}$. The fluorescence emission of zinc oxide nanoparticle shows high selectivity towards picric acid (PA) and can be used as a fluorescent probe for the detection of PA via fluorescence quenching mechanism. The fluorescence quenching mechanism of picric acid may be due to the electron transfer process between ZnO NPs and picric acid which is confirmed by cyclic voltammetry (CV). The quenching efficiency of nanoparticle was calculated using Stern-Volmer equation. The limit of detection was found to be $1.83 \mu \mathrm{M}$ for picric acid. The fluorescence property of the ZnO NPs can be utilized for cell imaging application as biosensors and also in the field of drug delivery.

Keywords: Boswellia ovalifoliolata; Zinc oxide nanoparticle; Picric acid; Nitroaromatic compound

\section{INTRODUCTION}

The development of sensitive and selective methods for the detection of nitroaromatics (NACs) explosives has been a field of enormous interest to researchers ${ }^{1}$. Picric acid is highly water soluble, strong organic acid and it is widely used as a powerful explosive like other NACs and is also a non-biodegradable environmental pollutant which causes skin and eye irritation, cancer, gastritis, aplastic anemia and liver damage $\mathrm{e}^{2,3}$. As compare with other NACs detection of picric acid (PA) is more important because of usage in the manufacture of rocket fuels, dye industries, fireworks, pharmaceuticals, deadly explosives, components in matches and chemical laboratories ${ }^{4,9}$. Picric acid is more dangerous in the dehydrated state, which can easily undergo spontaneous detonation to cause an explosion and it is more explosive than trinitrotoluene (TNT). In spite of all the awareness, PA has been released to the environment during explosives manufacturing, loading and in military installation for packing activities ${ }^{3,4}$. Different methods have been employed for the detection of NACs such as ionic liquids, $\mathrm{X}$-ray diffraction, organic gels, neutron activation, Raman spectroscopy, electrochemical, metal-organic frameworks, nanoparticle, polymers, quantum dots, ion mobility in solution and vapor phase which are high cost effective and are not easily accessible $e^{5,6,12}$. Hence, fluorescence quenching based sensing is widely used and proven to be highly sensitive ${ }^{6}$, selective ${ }^{26}$, fast, low $\operatorname{cost}^{1}$, and simple instrumentation $^{6}$.

Plant mediated synthesis of metal nanoparticle is gaining an advantage over physical and chemical method because it is one of the biocompatible, nontoxic, eco-friendly, low cost and simplicity in methods ${ }^{17}$, wherein the chemical method uses the organic solvent, toxic reducing and chelating agent leads to threatening of the environment in various fields ${ }^{18}$. The high-water solubility of nanoparticle has a various application due to its biocompatible and non-toxic stabilizers. Among all the metal oxide nanoparticles, many research groups are focusing on ZnO NPs for the various applications like sensors, coating, cosmetics, environmental protection, biology ${ }^{19}$, electronics, communication and medicinal industry along with its biological application like biological sensing, labelling, gene delivery, drug delivery and nanomedicine ${ }^{20}$. A few reports are available on the luminescent sensors of zinc oxide nanoparticles ( $\mathrm{ZnO}$ NPs) for the detection of metal ions and detection of organic molecules ${ }^{34}$. Muk et al. reported on luminescent $\mathrm{ZnO}$ NPs as $\mathrm{Cu}^{2+}$ sensors by quenching mechanism. Sharma et al. obtained imine linked $\mathrm{ZnO}$ NPs for $\mathrm{Co}^{2+}$ sensor ${ }^{8}$. The imine linked receptor coated on the ZnO NPs is responsible for the $\mathrm{Co}^{2+}$ sensing ${ }^{9}$. Zhao et al. have studied the APTES capped luminescent $\mathrm{ZnO}$ NPs for the selective and sensitive detection of dopamine by quenching of their luminescent ${ }^{10}$. Singh et al. synthesized water stable APTES capped $\mathrm{ZnO}$ NPs for the selective and sensitive detection of PA ${ }^{11}$.

Boswellia ovalifoliolata (N.P.Balakr. \& A.N.Henry) belongs to the family burseraceae. It is a habitat of seshachalam hill range of the eastern ghats of India. The plant is widely used by the tribal people like nakkala, sugali, chenchu to treat a number of ailments. Leaf, gum, and stem bark extract of the plant found to be anti-inflammatory, analgesic, cardiotoxicity ${ }^{21}$ cytotoxic $^{22}$ and hepatoprotective 
activity $^{23}$.

Herein, we report the green synthesis of $\mathrm{ZnO}$ nanoparticle using Boswellia ovalifoliolata extract and synthesized NPs were characterized using UV-visible (UV-vis) spectroscopy, X-ray diffraction (XRD), Fourier transformed infrared spectroscopy (FT-IR). Morphology of the synthesized NPs was examined by scanning electron microscopy (SEM) and the fluorescence property of NPs was measured using fluorescence spectroscopy (FL). The synthesized NPs exhibits strong fluorescent emission effect which can act as a chemo sensor for detection of PA which is a powerful explosive among other NACs. To the best of our knowledge, there is no literature available for the detection of picric acid by $\mathrm{ZnO}$ nanoparticle using plant extract for the synthesis of nanoparticle by greener approach. Thus, the synthesized $\mathrm{ZnO}$ nanoparticle is highly selective, sensitive, low cost and efficient in sensing picric acid.

\section{EXPERIMENTAL}

\subsection{Materials}

Boswellia ovalifoliolata (BO) was collected from seshachalam hills of Andhra Pradesh during the month of February 2015 and authenticated by Plant Anatomy Research Centre, Chennai (PARC/2015/3202). Zinc acetate was purchased from Sigma Aldrich whereas sodium hydroxide $(\mathrm{NaOH})$, picric acid (PA), 4-nitrophenol (NP), 2,4-dinitrophenol (DNP), 3-nitrobenzoic acid (NBA), 2,4-dinitrotoluene (DNT) were obtained from SD Fine-Chem Limited. 1,3-dinitro benzene (DNB), 4-nitrobenzaldehyde (BAO), nitrobenzene (NB), 4-nitrotoluene (NT) were purchased from Avra Laboratories private limited.

\subsection{Methods}

\subsubsection{Preparation of B. ovalifoliolata bark extract}

About $5 \mathrm{~g}$ of B.ovalifoliolata bark were used for synthesis of $\mathrm{ZnO}$ nanoparticles. The bark powder was dissolved in $100 \mathrm{ml}$ of distilled water and transferred into an Erlenmeyer flask boiled at $80{ }^{\circ} \mathrm{C}$ for $30 \mathrm{~min}$. The bark extract was filtered through Whatmann No.1 filter paper and the material was stored at $4{ }^{\circ} \mathrm{C}$ for the further use. The obtained bark extract (pH 6.0) will control size and shape of the nanoparticle. It will also act as a stabilizer for NPs synthesis ${ }^{19,20,24,25}$.

\subsubsection{Green synthesis of ZnO NPs using B. ovalifoliolata bark extract}

About $10 \mathrm{ml}$ of bark extract was mixed with $90 \mathrm{ml}$ of $5 \mathrm{mM}$ zinc acetate in aqueous solution with continuous stirring using a magnetic stirrer at $37{ }^{\circ} \mathrm{C}$ for $1 \mathrm{~h}$. The precursor and bark extract composition 1:9 volume ratio was prepared for the synthesis. After $1 \mathrm{~h}, 1 \mathrm{M}$ sodium hydroxide solution was added in drops to maintain the $\mathrm{pH} 8$ and kept for continuous stirring for $1 \mathrm{~h}$. The yellowish white colour precipitate was centrifuged at 4,000 rpm for $20 \mathrm{~min}$ and the precipitate was washed with ethanol for three times to remove impurities present in the final product and the powder will be dried in an oven at $100{ }^{\circ} \mathrm{C}$ for $5 \mathrm{~h}^{19,20,24}$.

\subsubsection{Characterization of NPs}

The formation of NPs is confirmed by UV-visible absorption spectra measured by a JASCO V-670 spectrophotometer at room temperature wavelength range between 200-800 nm. The FTIR of the bark extract and NPs were recorded by a SHIMADZU Infrared spectrophotometer (400-4000 $\mathrm{cm}^{-1}$ ) resolution IV. Fluorescence spectra were measured using Hitachi F-7000FL spectrometer with $150 \mathrm{~W}$ Xe lamp as excitation source. The surface morphology and particle size of the synthesized nanoparticles were examined using SEM using Carl Zeiss EVO 18, Germany and X-ray diffraction (XRD) method using Bruker D8 advanced through Ni-filtered $\mathrm{Cu} \mathrm{K} \alpha(\lambda=0.15406 \mathrm{~nm})$ with the range of $2 \theta=$ 10-90. High-Resolution Transmission electron microscope (HR-TEM) were recorded by using Transmission Electron Microscopy (TEM), (Model JSM 6390 LV, JOEL, USA). A drop of ZnO NPs was coated over the surface of the copper grid and the grid was dried. For SEM analysis, the samples were coated in cover slips.

\subsubsection{Cyclic voltammetry analysis}

The electrochemical study was carried out using cyclic voltammetry at different scan rate. A three-electrode system consists of glassy carbon electrode as working electrode (WE), platinum counter electrode and saturated silver and silver chloride as reference electrode (RE). $0.1 \mathrm{M}$ of Tetrabutylammonium tetrafluoroborate (TBAF) was used as a supporting electrolyte for the analysis.

\subsubsection{Fluorescence microscopy}

Fluorescence microscopy was used to evaluate the bioimaging application of the ZnO NPs and to assess the labelling capability of the nanoparticle. The nanoparticle was prepared and suspended in PBS pH 7.4 (0.1 M) and the nanoparticle fluorescence images were recorded using Fluorescence microscope (FM 3000, Wesmox, India).

\section{RESULTS AND DISCUSSION}

ZnO nanoparticle was synthesized using Boswellia ovalifoliolata extract due to the higher content of polyphenol may be responsible for the ZnO NPs formation. The aromatic hydroxyl presents in the Boswellia ovalifoliolata interact with zinc ions to form the complex in acidic medium and the precipitate formation started at $\mathrm{pH} 8.0^{18,23}$. The presence of phytochemicals in the extract like polyphenols, terpenoids tannins, anthocyanins and steroids act as a capping agent in the formation of $\mathrm{ZnO}$ NPs.

The prepared ZnO NP were characterized by X-ray diffraction pattern to confirm crystallinity and phase formation of the nanoparticle. XRD pattern of the sample were shown in Figure 1 and the detected peaks corresponds to the hexagonal phase in the lattice planes of (100), (002), (101), (102), (110), (103), (200), (112) and (201) shows $2 \theta$ values at $31.7^{\circ}, 34.48^{\circ}, 36.18^{\circ}, 47.52^{\circ}, 56.53,63.01^{\circ}$, $66.33^{\circ}, 68.09^{\circ}$ and $68.94^{\circ}$. The crystallinity of sample with narrow peak confirmed the ZnO NPs formation. The morphology and size were observed using scanning electron microscope ${ }^{20}$. The average crystallite size of the synthesized nanoparticle was found to be $17.7 \mathrm{~nm}$. It is noteworthy, compare to chemical method Figure S1 (43.51 $\mathrm{nm})$, nanoparticle synthesized using plant extract showed 


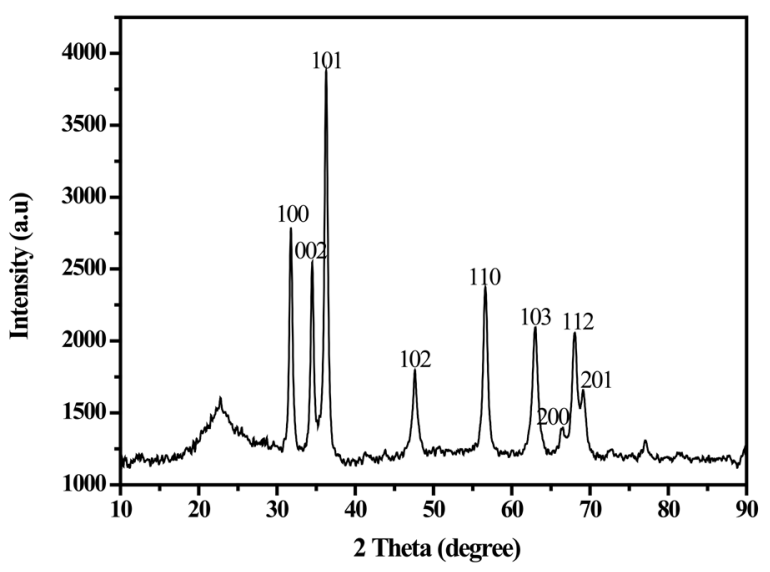

Figure 1. XRD pattern of $\mathrm{ZnO}$ nanoparticle synthesized from Boswellia ovalifoliolata extract.

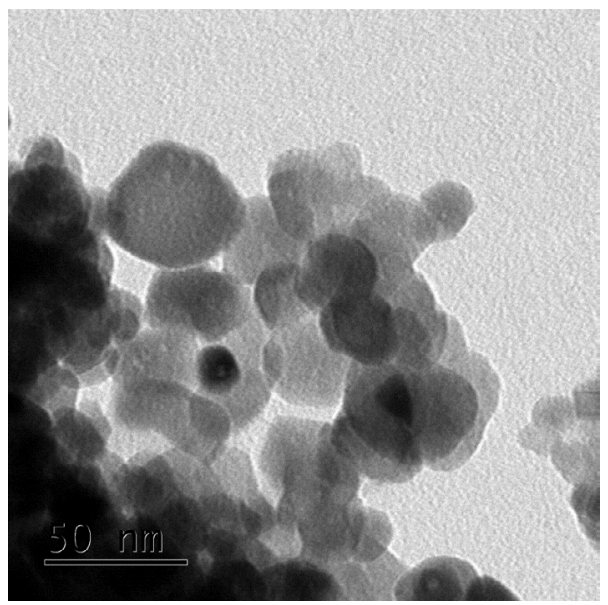

Figure 2. TEM images of $\mathrm{ZnO}$ nanoparticle in $50 \mathrm{~nm}$.

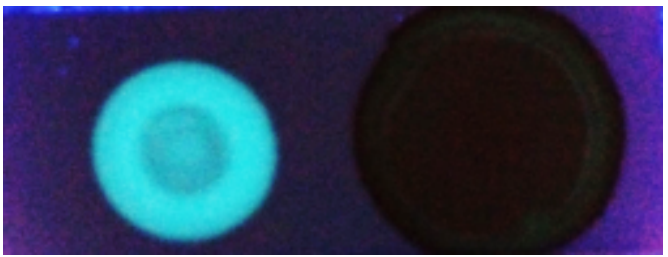

Figure 3. Fluorescence image (under $365 \mathrm{~nm}$ UV light) of a) $\mathrm{ZnO}$ nanoparticle adsorbed on a TLC plate and b) $\mathrm{ZnO}$ nanoparticle with a spot of PA solution.

reduced and control over size. The plant extract use to control the size of the nanoparticle and act as a stabilizing agent $^{21}$.

\subsection{FTIR analysis of ZnO NPs}

The FT-IR spectra of ZnO NPs-picric acid, ZnO NPs and extracts are shown in Figure S2. The chemical structure of the synthesized compound is obtained in the region of 4000$400 \mathrm{~cm}^{-1}$. ZnO NPs- picric acid showed prominent Ar- $\mathrm{NO}_{2}$ bands at 1568 and $1363 \mathrm{~cm}^{-1}$. The bands at 3523-3326 $\mathrm{cm}^{-1}$ correspond to $\mathrm{O}-\mathrm{H}$ mode of vibration ${ }^{18}$. We observed an alkyl $\mathrm{C}-\mathrm{H}$ peak at $2858 \mathrm{~cm}^{-1}$. The $\mathrm{ZnO}$ peak appears in the range of $530 \mathrm{~cm}^{-1}$ confirming the $\mathrm{ZnO}$ formation ${ }^{20,24}$. The bands of aromatic compounds around 757, 902 and $1063 \mathrm{~cm}^{-1}$ corresponds to stretching of aromatic $\mathrm{C}-\mathrm{H}$ and stretching of alcoholic $\mathrm{C}-\mathrm{O}$. Figure S2 shows a new peak at $1715 \mathrm{~cm}^{-1}$ of $\mathrm{C}=\mathrm{O}$ stretching in $\mathrm{ZnO}$-picric acid ${ }^{25,35}$. The peaks intensity of $\mathrm{ZnO}$-Picric acid was relatively reduced than $\mathrm{ZnO}$ NPs which may be due to the quenching effect of picric acid by removal of the organic compound from $\mathrm{ZnO} \mathrm{NPs}^{25}$. The nanoparticle is stabilized by the organic compound in the plant extract by the surface bound by their organic compounds. The phenolic compounds in the plant extract had a tendency to coordinate with zinc ions, thus stabilizing and preventing $\mathrm{ZnO}$ nanoparticle from being agglomerated. The results confirm that bioactive compounds were absorbed on the surface of $\mathrm{ZnO}$ nanoparticles. In this study, Boswellia ovalifoliolata extract was used for the synthesis, capping and chelating agents of nanoparticles.

\subsection{Transmission Electron Microscopy (TEM) analysis}

ZnO NPs was dispersed in ethanol in a carbon coated copper grid and the TEM image of ZnO NPs is shown in Figure 2. The HR-TEM image indicates that the ZnO NPs are slightly agglomerated and spherical in shape. The average particle size of the synthesized nanoparticle was found to be $11.6 \pm 4 \mathrm{~nm}$.

\subsection{Photo physical properties of ZnO NPs}

The photo physical properties of ZnO NPs using UV-visible spectra in aqueous solution were measured. Similarly, the fluorescence spectra were recorded in the range between 200-450 $\mathrm{nm}$. ZnO NPs in aqueous solution shows maximum absorption bands at $278 \mathrm{~nm}$ and fluorescence emission at $317 \mathrm{~nm}$. The low fluorescence emission of the $\mathrm{ZnO}$ nanoparticle may be due to the intrinsic defects produced by the $\mathrm{ZnO}$ nanoparticle such as interstitial zinc and oxygen vacancy and these defects will affect the fluorescence behaviour of the nanoparticle ${ }^{14,15}$. The high-energy absorption band in the region $278 \mathrm{~nm}$ can indicate the $\pi-\pi^{*}$ electron transition of phytochemicals in the plant extract such as phenols, tannins, steroids and terpenoids ${ }^{12,13}$. The photo physical property of the ZnO NP using UV-vis and fluorescence spectroscopy techniques aqueous solution, concentration were maintained at $10 \mathrm{mg} / \mathrm{L}$. The green color fluorescence image of the nanoparticle adsorbed on a TLC plate are shown in Figure 3a. The green emission of the $\mathrm{ZnO}$ nanoparticle may be due to the oxygen vacancies located on the surfaces. The blue emission may be due to the zinc vacancies ${ }^{14,15}$.

\subsection{Scanning electron microscopy (SEM)}

The surface morphological nature of the ZnO NPs is investigated using SEM techniques. The SEM images of ZnO NPs showed agglomerated nanoparticle Figure $4 a^{7,16,27}$. Addition of PA leads to a microstructural changes. The addition of PA molecules leading to the formation of irregular square like structure with length of about $2 \mu \mathrm{m}$ Figure $4 \mathrm{~b}$ of the SEM image allows the migration of excitons in a large number of channels which ultimately results in sensitivity towards picric acid. The elemental composition of $\mathrm{ZnO}$ nanoparticle obtained from EDAX analysis (Figure 4c) was Zinc (48.52\%), Oxygen (28.83\%) and Carbon (22.65\%). The presence of carbon indicates the connection of plant phytochemicals in size reduction and in capping of the synthesized $\mathrm{ZnO} \mathrm{NPs}^{18}$.

\subsection{Detection of picric acid (PA)}



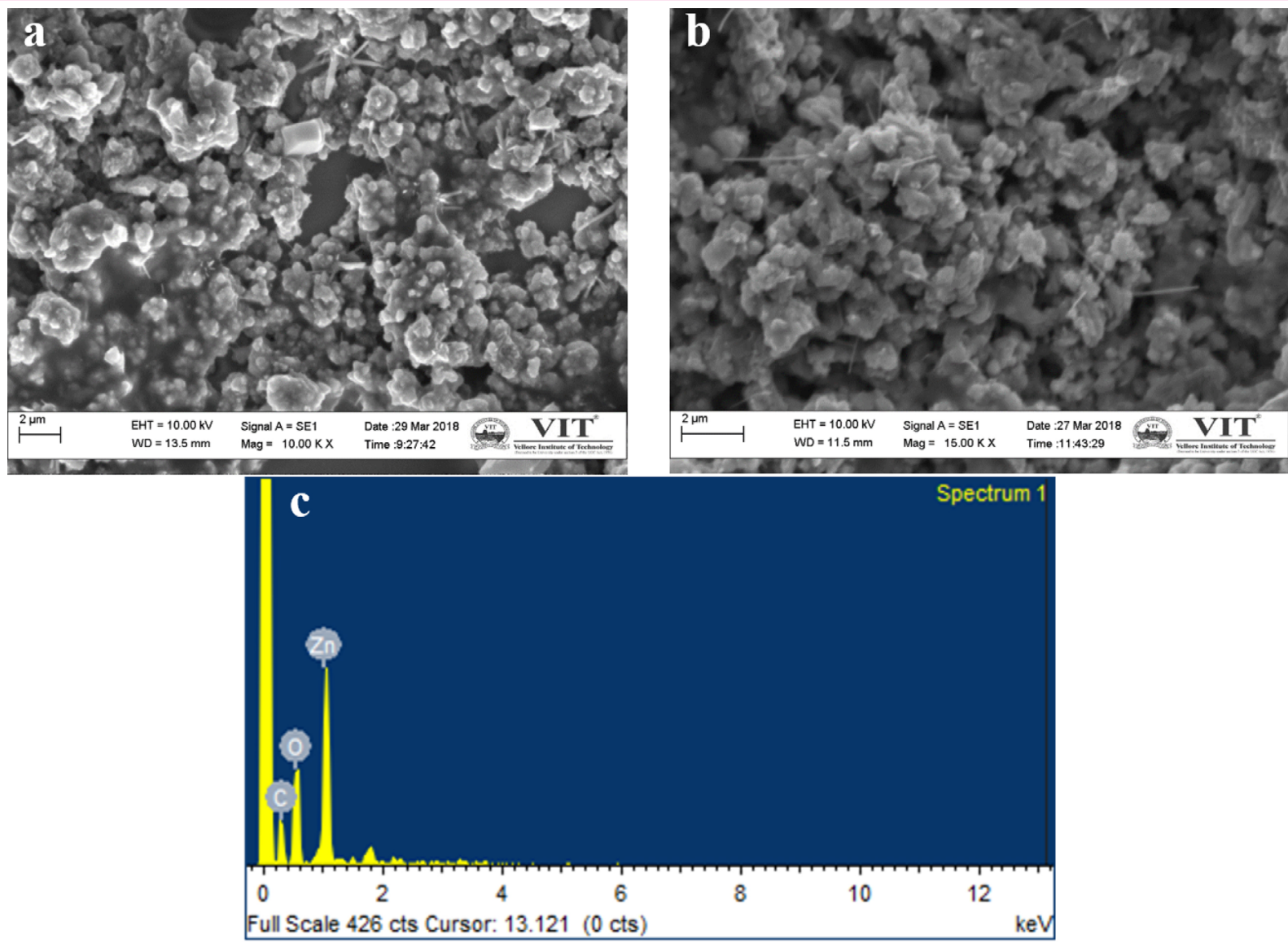

Figure 4. Scanning electron microscopy (SEM) images of a) ZnO nanoparticle, b) after PA addition and c) EDAX spectrum of $\mathrm{ZnO}$ nanoparticle.

The strong emission of nanoparticle in aqueous solution encouraged us to explore their possible application as chemosensor for the detection of nitroaromatic compounds such as picric acid (PA), dinitrotoluene (DNT), nitrobenzene (NB), nitrophenol (NP), 4-nitrobenzaldehyde (BAO), dinitrobenzene (BNB), dinitrophenol (DNP), 4-nitrobenzoic acid (NBA) and nitrotoluene (NT). The detection of nitroaromatic compounds has attracted many researchers to fight against terrorism and environmental impact ${ }^{26}$. A number of techniques used for the detection of nitroaromatic compounds, but fluorescence-based detection offers many advantages like high sensitivity, short response time and specificity ${ }^{1}$. The fluorescence based detection of NACs in presence of $\mathrm{ZnO}$ NPs as the chemo sensor was performed by using UV-vis and fluorescence titrations and the analytes were taken in very low concentration. To evaluate the selectivity of ZnO NPs towards PA, the fluorescence quenching efficiency of different nitro compounds were investigated. Initially, the UV-vis titration of ZnO NP with the NACs and the spectra obtained are shown in Figure S3. The UV-vis absorption spectra of ZnO NPs increase for specific analyte PA. The UV-vis titration of NPs was carried out for PA is shown in Figure S4 and the absorbance was gradually increased with the addition of PA.

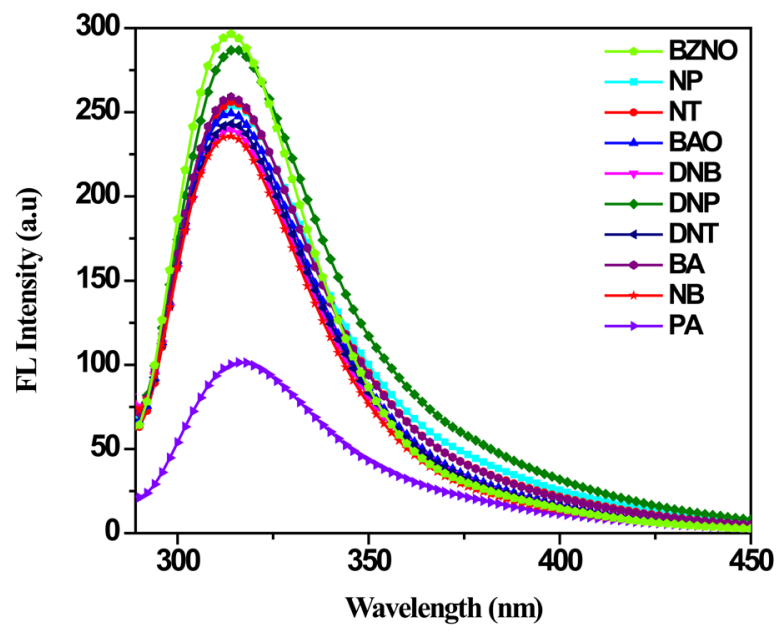

Figure 5. Fluorescence emission spectra of $\mathrm{ZnO}$ nanoparticle with different nitroaromatics.

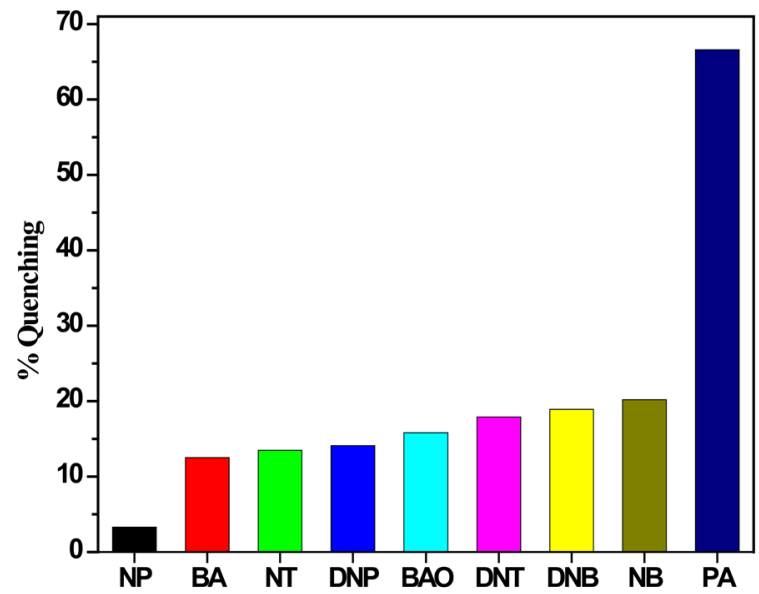

Figure 6. Fluorescence quenching of $\mathrm{ZnO}$ nanoparticle with the addition of different nitroaromatics. 


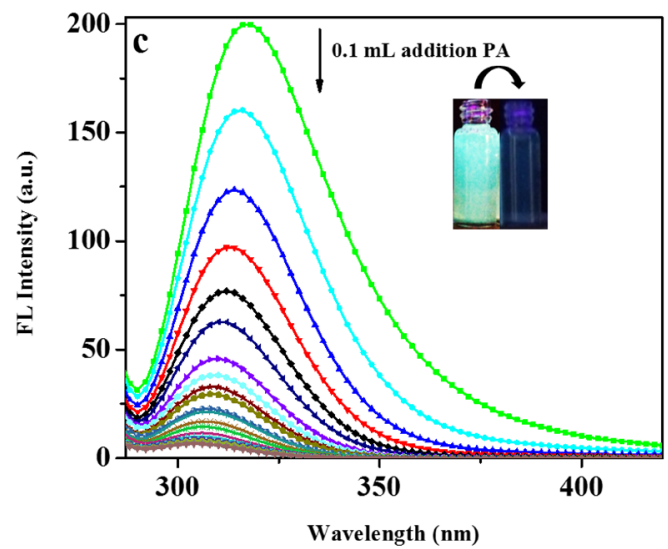

Figure 7. Fluorescence emission spectra of ZnO NPs in aqueous solution with PA titration.

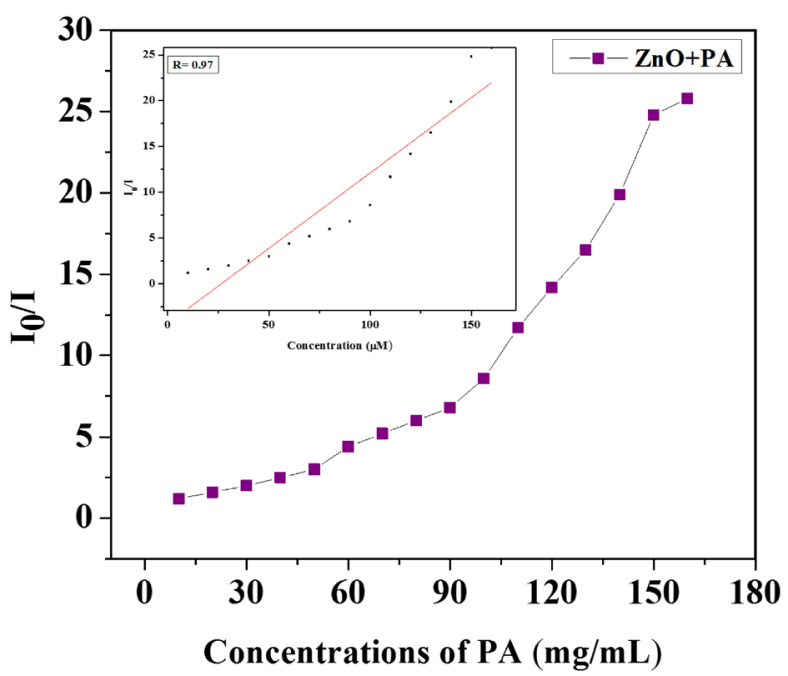

Figure 8. Stern-Volmer plot of ZnO NPs in aqueous solution with the addition of picric acid. Inset shows the relative fluorescence intensity with concentration up to 100 $\mu \mathrm{M}$.

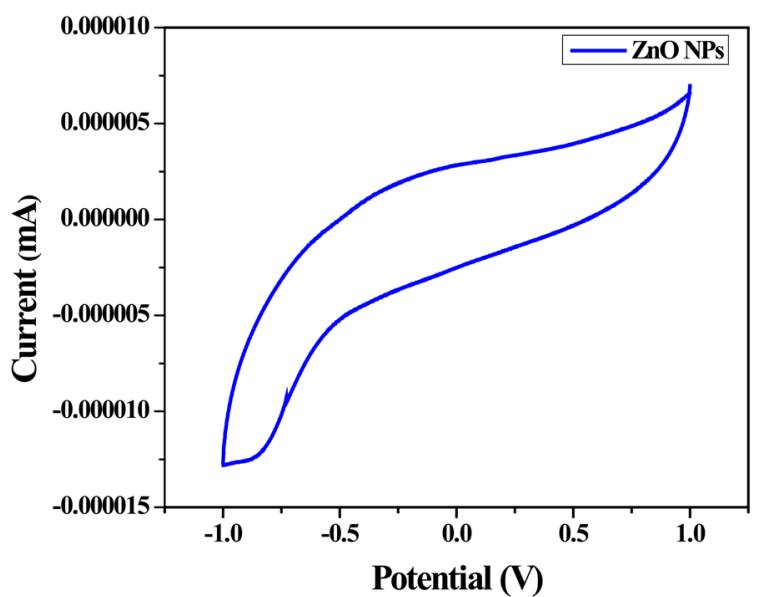

Figure 9. Cyclic voltammetry of ZnO NPs in water as solvent a scan rate at $100 \mathrm{mV} \mathrm{s}^{-1}$.

The fluorescence emission spectra of ZnO NPs with different NACs are displayed in Figure 5. Among all the NACs picric acid showed best quenching efficiency, the decrease in fluorescence intensity indicates the quenching efficiency of PA and the percentage fluorescence quenching intensities are shown in Figure 6. Among the various nitroaro- matic compounds tested, the addition of picric acid to the solution of $\mathrm{ZnO}$ NPs in aqueous solution results in $70 \%$ quenching of fluorescence emission Figure $7^{2,7}$.

We found that the fluorescence property of $\mathrm{ZnO}$ nanoparticle can easily be quenched by PA, which allows us to develop a sensor for the detection of PA. PA showed more quenching efficiency than other explosives. The fluorescence of the ZnO NPs was partially quenched by other NACs, the quenching efficiency was much lesser than that of PA. In general, nitro aromatic compounds are nitro substituted electron acceptor molecules, they can bind to $\pi$-donor sites by donor-acceptor interactions. Fluorescence property of most electron-rich fluorophores compounds can be effectively quenched by nitro-aromatic compounds through electron transfer. Many hydroxyl groups are on the surface of ZnO NPs. Hence, when electron-deficient PA is added to ZnO NP solution, it may possibly be bound to the electron rich hydroxyl group present on the surface of $\mathrm{ZnO}$ NPs which causes quenching of fluorescence property of the solution where the excited electron is trapped by electron transfer mechanism ${ }^{5,27,29}$. The fluorescence quenching image of picric acid adsorbed on a TLC plate was shown in Figure 3b. A linear calibration curve of quenching efficiency with concentration of PA (up to $100 \mu \mathrm{M}$ ) is observed with correlation of coefficient $(\mathrm{R}=0.97)$ (Inset of Figure 8). The fluorescence quenching efficiency of $\mathrm{ZnO}$ NPs was studied using Stern-Volmer plot presented in Figure 8 equation $\left(\mathrm{I}_{0} / \mathrm{I}\right.$ vs [Q] where, $\mathrm{I}_{0}$ represents fluorescence intensity in the absence of quencher (PA). Fluorescence intensity was denoted in the presence of various concentration of picric acid. $\mathrm{Q}$ is the concentration of quencher.) By using the plot in Figure 8, the detection limit was found to be $1.83 \mu \mathrm{M}$ for ZnO NPs using the equation $3 \sigma /$ slope. Further, to study the electron transfer process in the quenching mechanism of picric acid, the highest occupied molecular orbital (HOMO) level and lowest unoccupied molecular orbital (LUMO) level of ZnO NPs was obtained using cyclic voltammetry studies Figure 9. Figure S5 shows the energy levels for ZnO NPs and PA. The onset oxidation peak potential of ZnO NPs was observed at $0.76 \mathrm{~V}$ subsequently the onset reduction peak potential was observed at $-0.46 \mathrm{~V}$. The HOMO energy level was calculated from onset oxidation potential using the following equation $\mathrm{HOMO}=-\mathrm{eV}\left(\mathrm{E}_{\mathrm{ox}}{ }^{\text {on- }}\right.$ set $\left.-\mathrm{E}_{1 / 2}{ }^{\text {ferrocene }}+4.8\right)$ and $\mathrm{LUMO}=-\mathrm{eV}\left(\mathrm{E}_{\text {red }}{ }^{\text {onset }}-\mathrm{E}_{1 / 2}{ }^{\text {ferrocene }}+\right.$ 4.8) respectively. The electron transfers from the $\mathrm{ZnO}$ NPs with $4.89 \mathrm{eV}$ (HOMO) and 3.66 (LUMO) values may result in the quenching process. The energy gap $(0.7 \mathrm{eV})$ between the HOMO of ZnO NPs $(4.89 \mathrm{eV})$ and the LUMO of PA $(4.19 \mathrm{eV})$ was lesser than the energy gap $(1.2 \mathrm{eV})$ between the HOMO (4.89 eV) and LUMO (3.66 eV) of ZnO NPs. The HOMO and LUMO levels of ZnO NPs were compared with the reported PA HOMO and LUMO energy levels. The fluorescence quenching of ZnO NPs may be due to the energy transfer between PA and $\mathrm{ZnO} \mathrm{NPs}^{27,30,31}$.

\subsection{Reproducibility}

The reproducibility of the ZnO NPs is the ability to produce the same responses for 5 times and the response has been measured Figure 10. Relative standard deviation was found to be $1.76 \%$. The relative quenching of fluorescence intensity of $\mathrm{ZnO}$ NPs in the presence of $100 \mu \mathrm{M}$ PA is 


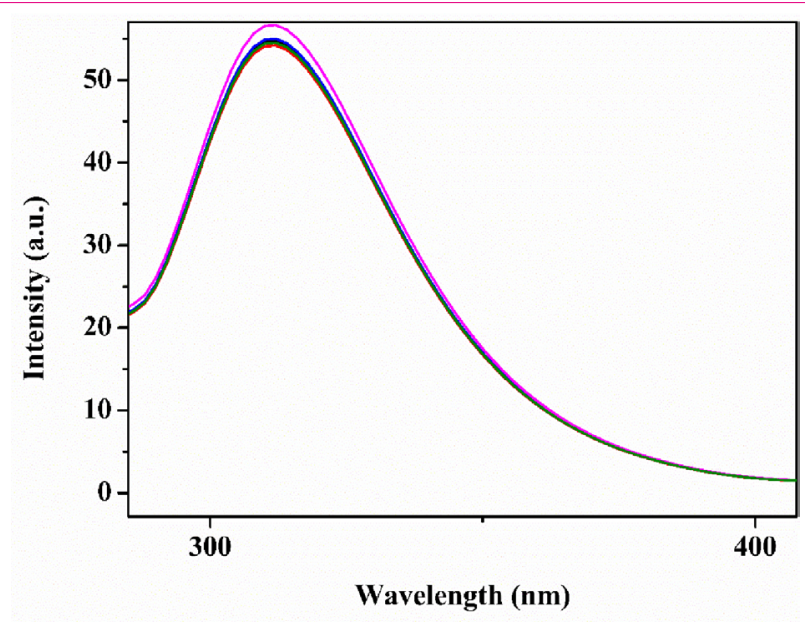

Figure 10. Reproducibility of ZnO NPs with $100 \mu \mathrm{M}$ of PA.
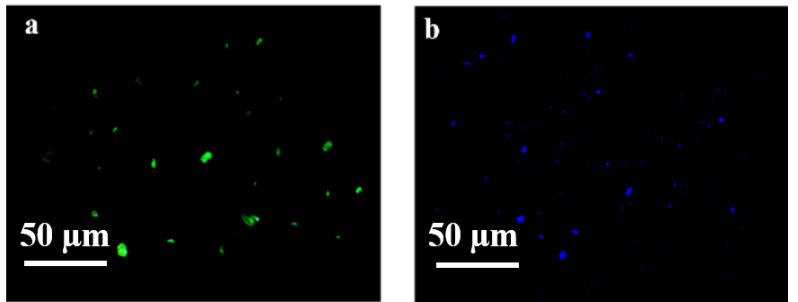

Figure 11. Fluorescence microscopy images of $\mathrm{ZnO}$ nanoparticle dispersed in PBS solution and the image was taken in a) green and b) blue filter.

$163.29 \pm 1.76 \%$.

\subsection{Fluorescence images of $\mathrm{ZnO}$ NPs}

The fluorescence nature of the ZnO NPs can be utilized for the bioimaging application. The NPs were treated with $\mathrm{PBS}^{33}$ to evaluate the fluorescence property of the particle and the prepared sample was placed on a microscope slide to acquire the fluorescence image. As shown in Figure 11, Unexpectedly the ZnO NPs exhibited blue fluorescence and green fluorescence. The photoluminescence measurements were conducted using long pass filters ${ }^{32}$. The obtained results indicate that $\mathrm{ZnO}$ NPs can also act as bioimaging probes or its application.

\section{CONCLUSION}

In conclusion, we synthesized fluorescent $\mathrm{ZnO}$ NPs with enhanced fluorescence using Boswellia ovalifoliolata extract. The ZnO NPs exhibited high selectivity, sensitivity, low cost and efficient in sensing picric acid. The fluorescenct property of $\mathrm{ZnO}$ NPs in aqueous media makes it more potential and can be used as a fluorescent sensor for detection of picric acid and also in bioimaging application. Further, the biocompatibility of the nanoparticle needs to be studied for the cellular imaging and biotechnology application. Hence, the synthesized $\mathrm{ZnO}$ nanoparticle can be utilized effectively for sensing picric acid.

\section{Acknowledgements}

The authors are thankful to VIT University for the financial support and facilities provided to us. Authors acknowledge Dr. G. Sathiyan for the valuable suggestions and discussions for this paper. We thank Prof. K. Suthindhiran and his student R. Varalakshmi for the kind help with the fluorescence microscopy image. We thank Ms K. Shalinidevi for giving support for the cyclic voltammetry analysis.

\section{Notes}

The authors declare no competing financial interest.

\section{References}

[1] Mahendran V, Shanmugam S. Aggregates of a hydrazono-sulfonamide adduct as picric acid sensors. RSC Advances. 2015;5(112):92473-9. doi:10.1039/c5ra17359k

[2] Pati PB, Zade SS. Highly emissive triphenylamine based fluorophores for detection of picric acid. Tetrahedron Letters. 2014;55(38):5290-3. doi:10.1016/j.tetlet.2014.07.098

[3] Mazumdar P, Maity S, Shyamal M, Das D, Sahoo GP, Misra A. Proton triggered emission and selective sensing of picric acid by the fluorescent aggregates of 6,7-dimethyl-2,3-bis-(2-pyridyl)-quinoxaline. Physical Chemistry Chemical Physics. 2016;18(10):705567. doi: $10.1039 / \mathrm{c} 5 \mathrm{cp} 05827 \mathrm{a}$

[4] Acharyya K, Mukherjee PS. A fluorescent organic cage for picric acid detection. Chem Commun. 2014;50(99):15788-91. doi:10.1039/c4cc06225f

[5] Chakravarty S, Gogoi B, Sen Sarma N. Fluorescent probes for detection of picric acid explosive: A greener approach. Journal of Luminescence. 2015;165:6-14. doi:10.1016/j.jlumin.2015.04.006 [6] Wang X, Guo Y, Li D, Chen H, Sun R. Fluorescent amphiphilic cellulose nanoaggregates for sensing trace explosives in aqueous solution. Chemical Communications. 2012;48(45):5569. doi:10.1039/c2cc30208j

[7] Bhalla V, Gupta A, Kumar M. Fluorescent Nanoaggregates of Pentacenequinone Derivative for Selective Sensing of Picric acid in Aqueous Media. Organic Letters. 2012;14(12):3112-5. doi:10.1021/ol301202v

[8] Ng SM, Wong DSN, Phung JHC, Chua HS. Integrated miniature fluorescent probe to leverage the sensing potential of $\mathrm{ZnO}$ quantum dots for the detection of copper (II) ions. Talanta. 2013;116:514-9. doi:10.1016/j.talanta.2013.07.031

[9] Sharma H, Singh A, Kaur N, Singh N. ZnO-Based ImineLinked Coupled Biocompatible Chemosensor for Nanomolar Detection of $\mathrm{Co}^{2+}$. ACS Sustainable Chemistry \& Engineering. 2013;1(12):1600-8. doi:10.1021/sc400250s

[10] Zhao D, Song H, Hao L, Liu X, Zhang L, Lv Y. Luminescent $\mathrm{ZnO}$ quantum dots for sensitive and selective detection of dopamine. Talanta. 2013;107:133-9. doi:10.1016/j.talanta.2013.01.006

[11] Singh K, Chaudhary GR, Singh S, Mehta SK. Synthesis of highly luminescent water stable $\mathrm{ZnO}$ quantum dots as photoluminescent sensor for picric acid. Journal of Luminescence. 2014;154:148-54. doi:10.1016/j.jlumin.2014.03.054

[12] Sathiyan G, Sakthivel P. A multibranched carbazole linked triazine based fluorescent molecule for the selective detection of picric acid. 2016;6(108):106705-15. doi:10.1039/c6ra22632a

[13] Sathiyan G, Balasubramaniam B, Ranjan S, Chatterjee S, Sen P, Garg A, et al. A novel star-shaped triazine-triphenylaminebased fluorescent chemosensor for the selective detection of picric acid. Materials Today Chemistry. 2019;12:178-86. doi:10.1016/ j.mtchem.2019.01.002

[14] Tan TL, Lai CW, Abd Hamid SB. Tunable Band Gap Energy of Mn-Doped ZnO Nanoparticles Using the Coprecipitation Technique. Journal of Nanomaterials. 2014;2014:1-6. doi:10.1155/2014/371720

[15] Zhang Z-Y, Xiong H-M. Photoluminescent ZnO Nanoparticles 
and Their Biological Applications. Materials. 2015;8(6):3101-27. doi:10.3390/ma8063101

[16] Hong Y, Lam JWY, Tang BZ. Aggregation-induced emission: phenomenon, mechanism and applications. Chemical Communications. 2009;(29):4332. doi:10.1039/b904665h

[17] Yi X, Li J, Zhu Z, Liu Q, Xue Q, Ding D. In vivo cancer research using aggregation-induced emission organic nanoparticles. Drug Discovery Today. 2017;22(9):1412-20. doi:10.1016/j.drudis.2017.04.004

[18] Santhoshkumar J, Kumar SV, Rajeshkumar S. Synthesis of zinc oxide nanoparticles using plant leaf extract against urinary tract infection pathogen. Resource-Efficient Technologies. 2017;3(4):459-65. doi:10.1016/j.reffit.2017.05.001

[19] Jin Y, Qian Y. Photophysical properties, aggregation-induced fluorescence in nanoaggregates and cell imaging of 2,5-bisaryl 1,3,4-oxadiazoles. New Journal of Chemistry. 2015;39(4):2872-80. doi:10.1039/c4nj02293a

[20] Tang F, Wang C, Wang J, Wang X, Li L. Fluorescent Organic Nanoparticles with Enhanced Fluorescence by Self-Aggregation and their Application to Cellular Imaging. ACS Applied Materials \& Interfaces. 2014;6(20):18337-43. doi:10.1021/am505776a

[21] Kuppusamy P, Yusoff MM, Maniam GP, Govindan N. Biosynthesis of metallic nanoparticles using plant derivatives and their new avenues in pharmacological applications - An updated report. Saudi Pharmaceutical Journal. 2016;24(4):473-84. doi:10.1016/j.jsps.2014.11.013

[22] Mittal AK, Chisti Y, Banerjee UC. Synthesis of metallic nanoparticles using plant extracts. Biotechnology Advances. 2013;31(2):346-56. doi:10.1016/j.biotechadv.2013.01.003

[23] Koupaei MH, Shareghi B, Saboury AA, Davar F, Semnani A, Evini M. Green synthesis of zinc oxide nanoparticles and their effect on the stability and activity of proteinase K. RSC Advances 2016;6(48):42313-23. doi:10.1039/c5ra24862k

[24] Bala N, Saha S, Chakraborty M, Maiti M, Das S, Basu R, et al. Green synthesis of zinc oxide nanoparticles using Hibiscus subdariffa leaf extract: effect of temperature on synthesis, anti-bacterial activity and anti-diabetic activity. RSC Advances. 2015;5(7):4993-5003. doi:10.1039/c4ra12784f

[25] Agarwal H, Venkat Kumar S, Rajeshkumar S. A review on green synthesis of zinc oxide nanoparticles - An eco-friendly approach. Resource-Efficient Technologies. 2017;3(4):406-13. doi:10.1016/j.reffit.2017.03.002

[26] Uma Mahesh B, Shrivastava S, Kuncha M, Sahu BD, Swamy CV, Pragada RR, et al. Ethanolic extract of Boswellia ovalifoliolata bark and leaf attenuates doxorubicin-induced cardiotoxicity in mice. Environmental Toxicology and Pharmacology. 2013;36(3):840-9. doi:10.1016/j.etap.2013.07.016

[27] Thummuri D, Jeengar MK, Shrivastava S, Areti A, Yerra VG, Yamjala S, et al. Boswellia ovalifoliolata abrogates ROS mediated NF- $\kappa \mathrm{B}$ activation, causes apoptosis and chemosensitization in Triple Negative Breast Cancer cells. Environmental Toxicology and Pharmacology. 2014;38(1):58-70. doi:10.1016/j.etap.2014.05.002

[28] Mahesh BU, Shrivastava S, Pragada RR, Naidu VGM, Sistla R. Antioxidant and hepatoprotective effects of Boswellia ovalifoliolata bark extracts. Chinese Journal of Natural Medicines. 2014;12(9):663-71. doi:10.1016/s1875-5364(14)60101-1

[29] Alias SS, Ismail AB, Mohamad AA. Effect of pH on $\mathrm{ZnO}$ nanoparticle properties synthesized by sol-gel centrifugation. Journal of Alloys and Compounds. 2010;499(2):231-7. doi:10.1016/j.jallcom.2010.03.174

[30] Khan IM, Ahmad A, Oves M. Synthesis, characterization, spectrophotometric, structural and antimicrobial studies of the newly charge transfer complex of p-phenylenediamine with $\pi$ acceptor picric acid. Spectrochimica Acta Part A: Molecular and Biomolecular Spectroscopy. 2010;77(5):1059-64. doi:10.1016/ j.saa.2010.08.073

[31] Zhao L-H, Zhang J, Sun S-Q. Stable aqueous ZnO nanoparticles with green photoluminescence and biocompatibility. Journal of Luminescence. 2012;132(10):2595-8. doi:10.1016/j.jlu$\min .2012 .04 .028$

[32] Sandhu S, Kumar R, Singh P, Mahajan A, Kaur M, Kumar S. Ultratrace Detection of Nitroaromatics: Picric Acid Responsive Aggregation/Disaggregation of Self-Assembledp-Terphenylbenzimidazolium-Based Molecular Baskets. ACS Applied Materials \& Interfaces. 2015;7(19):10491-500. doi:10.1021/acsami.5b01970

[33] Sathiyan G, Thangamuthu R, Sakthivel P. Synthesis of carbazole-based copolymers containing carbazole-thiazolo[5,4-d] thiazole groups with different dopants and their fluorescence and electrical conductivity applications. RSC Advances. 2016;6(73):69196-205. doi:10.1039/c6ra08888k

[34] Liu SG, Luo D, Li N, Zhang W, Lei JL, Li NB, et al. Water-Soluble Nonconjugated Polymer Nanoparticles with Strong Fluorescence Emission for Selective and Sensitive Detection of Nitro-Explosive Picric Acid in Aqueous Medium. ACS Applied Materials \& Interfaces. 2016;8(33):21700-9. doi:10.1021/acsami.6b07407

[35] Malik AH, Hussain S, Kalita A, Iyer PK. Conjugated Polymer Nanoparticles for the Amplified Detection of Nitro-explosive Picric Acid on Multiple Platforms. ACS Applied Materials \& Interfaces. 2015;7(48):26968-76. doi:10.1021/acsami.5b08068

[36] Su Y, Shi W, Chen X, Zhao S, Hui Y, Xie Z. An aggregation-induced emission enhancement fluorescent benzoxazine-derived macromolecule: catalyst-free synthesis and its preliminary application for the determination of aqueous picric acid. RSC Advances. 2016;6(47):41340-7. doi:10.1039/c6ra06942h

[37] Kung M-L, Lin P-Y, Hsieh C-W, Tai M-H, Wu D-C, Kuo C-H, et al. Bifunctional Peppermint Oil Nanoparticles for Antibacterial Activity and Fluorescence Imaging. ACS Sustainable Chemistry \& Engineering. 2014;2(7):1769-75. doi:10.1021/sc500242k

[38] Xiong H-M. ZnO Nanoparticles Applied to Bioimaging and Drug Delivery. Advanced Materials. 2013;25(37):5329-35. doi:10.1002/adma.201301732

[39] Zhang Z-Y, Xiong H-M. Photoluminescent ZnO Nanoparticles and Their Biological Applications. Materials. 2015;8(6):3101-27. doi: $10.3390 / \mathrm{ma} 8063101$

\section{Open Access}

This article is licensed under a Creative Commons Attribution 4.0 International License.

๑) The Author(s) 2019 